\title{
Design of a Guide System for Motor Skill Development Targeting a Repetitive Movement Task
}

\author{
Kenji Matsuura*, Hiroshi Toyooka*, Stephen Karungaru* and Naka Gotoda**
}

(Received 8 June 2017 and accepted in revised form 19 December 2017)

\begin{abstract}
This article discusses a system designed for repetitive movement learning. The concrete target domain is a motor-skill, which requires both knowledge and physical ability. Cyclic behavior of a segmented unit constitutes repetitive movement. It is hard to learn a stabilized way of such movement without any guides. Therefore, the present study provides a supporting system based on a model for guiding players to make appropriate motor actions. Two types of functionality, which work with a fixed model and a fluctuation model, can be optionally selected. This paper mainly deals with the latter one, which needs monitoring of behavior and adjusting of the trigger for guiding from technical viewpoints. Through an experiment, significant improvements are found in performance although subjects do not recognize the difference consciously during practice.
\end{abstract}

Keywords: guide system, repetitive movement, skill development, motion capture, real time support

\section{Introduction}

Practicing something repeatedly is one of the major ways to acquire skills or knowledge such as basic arithmetic operations, second language conversation and other intellectual domains. Skill learning in physical domains also needs a kind of drilled practice. Motor skill is regarded as an ability coordinated with body movements in the real world to achieve a goal, which was previously set in the mind. According to the conceptual definition offered by Guthrie ${ }^{(1)}$, skill is the ability to bring about some end result with maximum certainty and minimum outlay of energy or of time and energy. In line with this concept, a learner tries to get used to demonstrating a pattern stored inside while attempting to strengthen the tried pattern and to increase the number of patterns. New patterns of movement are a challenge in a positive sense. In training, a well-organized manner with a theoretical basis is necessary.

Over time training results in an exact reproduction of some activity. Hence, Schmidt ${ }^{(2)}$ described a fundamental schema theory of how humans learn discrete perceptual motor skills and lots of studies have been done following the theory. According to publications about schema theory, coordinated movement is a combination of body movements together with adjustment of spatial direction, force and timing. Therefore, learning these

\footnotetext{
* Graduate School of Advanced Technology and Science,

Tokushima University, Japan

**Information Technology Center, Kagawa University, Japan
}

kinematic and kinetic parameters of an appropriate timing is regarded as the first stage in which learners acquire the skill through trial and error activities ${ }^{(3)}$. Johnson $^{(4)}$ stated that elements of form, accuracy, speed and adaptability provide a skill where these elements are linked to the timing of a motion trigger. The mechanism to support the trigger for starting a motion is more effective when it is on time rather than delayed. Hence, the present paper discusses a guiding issue of the motion trigger during the movement at first.

Several domains are regarded as a potential target of repetitive movements. Rope skipping ${ }^{(5)}$, swimming, running, sitting on a swing and other activities that can be imagined as repetitive movements have some timing triggers in the continuous motion. Our concrete target in this study is to support development of a hula-hoop twirling skill. It is a kind of gross motor skill, and its difficulty is in operating an object around the player's abdomen without applying pressure using the limbs. In addition, the wave form of a hoop trajectory sometimes indicates coherence consisting of plural and different periodic movements in both short and long periods. Therefore, we pick the domain of the target as a discussion topic. Regarding the basic movement in playing with a hula-hoop, the player has to control the height of the hoop by movement of her/his waist ${ }^{(6)}$. To prevent hula-hoop from falling down, the player has to recognize the movement of the hoop around the waist and the speed of the waist adjusting to the timing of the hoop movement. It is important that the player does not 
deflect the movement in the wrong direction.

Even for an expert, unwanted or unconscious perturbation occurs at every cycle of the hula-hoop trajectory. Most of the proposed applications by traditional control theory work on a target with delayed feedback to counteract the current movement or the position in an irrelevant situation. Delayed feedback for synchronous support does not work sufficiently because of the perturbation, offset effect and other factors. In general, a periodic guide of starting motion is tried but it is more effective when people do not recognize the object movement at all. However, haptic, visual and auditory senses always monitor the environment and that implies a time scale calibration is necessary. Learners are not aware of the difference between recognition and performance explicitly. Hence, this paper proposes a new model in order to support learning repetitive movement by a guiding method with predictive adjustment for the next motor timing.

\section{Background to the Proposed Model}

\subsection{Conventional Motor Control Model}

We start with a brief literature review. Schmidt ${ }^{(2)}$ claimed that the human process of a motor skill performance starts with input signals from an environment through some sense organs. There are several ways of processing internal information including stimulus identification, response selection and response programming. Output through those processes, which might seem as a sort of black box, proceeds to the motor program and its physical performance. Though the illustration of such human processes mainly deals with a onetime trial, how humans change behavior requires a slightly different aspect of learning.

Regarding learning stages of motor control, Fitts and Possner ${ }^{(7)}$, touched upon three major stages; cognitive, associative and autonomous. A learner in the cognitive stage learns or recognizes the movement control consciously. During the next stage, the learner tries to implement an obtained model with recognition on some body-parts movement consciously. At last in the autonomous stage, the movement way is integrated automatically.

Learning motor control requires feedback in former stages in order to adjust the next production of the movement; this was pointed out in an article by Soga et al. ${ }^{(8)}$. Feedback influences some positive aspects, including motivation, discriminability, standardization, and avoiding dependability on habitual feedback.

\subsection{Hula-hoop Structure}

A hula-hoop is made of plastic tubing. Its movement can be given by a mathematical description. If a robot is programmed sophisticatedly, it can reproduce the hula-hoop movement in a whirl vibration mode forcibly. Some studies have tackled elucidation of hula-hoop movements from an ideal context such in the contribution of Seyranian and Belyakov ${ }^{(9)}$, which discusses a mathematical model in the same domain. Taking the real-world into account, however, there are several uncertain parameters that have an influence, i.e. frictional force. In addition, even in a case where a learner knows the mathematical meaning of a differential equation, it is very hard to implement the performance following the equation. As a result, either simplification or approximation is one of the practical solutions against this kind of issue as Matsuura et al. have reported ${ }^{(10)}$.

If a hula-hoop learner receives affirmative feedback during the movement, the latest performance and its controlled trajectory are stored inside the learner as reinforcing memory. On the contrary, unfavorable feedback countermines the short-term memory of the latest performance if the learner gets a negative reproduction result. These adjustments in learning often occur in the middle stage according to the proposal by Fitts and Possner ${ }^{(7)}$. This is an associative stage in which the learner actually performs the target task by trial and error.

\subsection{Cyclic Motor Action}

A simplified model with an equation of motion is approximately regarded as a simple harmonic motion. Exciting force applied by the waist in this context is a kind of restoration. A sufficient amount of the force $(F)$ is able to make the movement distance of the hoop to close to the enforcing position.

$$
F=-K x
$$

where $K$ in Equation (2.1) is a constant of proportionality and $x$ indicates displacement. $x$ is determined by the hoop radius.

In order to make the hoop movement uniform, $K$ 
should be stable at each enforcing time. Under these conditions, the relationship among angular velocity $(\omega)$, period $(T)$ and mass of the hoop $(m)$ is represented in Equation (2.2).

$$
T=\frac{2 \pi}{\omega}=2 \pi \sqrt{\frac{m}{K}}
$$

In order to keep the hoop from $F$ viewpoint, $T$ is defined by $\omega$ and adjusted as (2.2).

If we represent movement of the waist in a simple harmonic motion, its cycle is identified by the time from local maximum to the next local maximum in the $Z$ coordinate. Therefore, every half period from the local maximum indicates the timing of enforcing approximately. The grading is defined as the duration of two periods of movement timing in this study.

The movement trajectory of the hoop can be drawn in a horizontal plane. However, from a three-dimensional viewpoint, movement trajectory is an important criterion to monitor vertically because the relative position of the centroid indicates the stability of the hoop. Therefore, a stable state in this study is detected as the vertical difference between centroids of both the waist and the hoop movements. This is the criterion of the spacing.

The above discussions imply that, first a learner must recognize the space between the body centroid and the hoop centroid. We call this the spacing degree, in the stimulus identification process. In parallel, the next enforcing timing with its grading is calculated accordingly. The latter phase includes response selection and response programming according to Schmidt. These processes are performed repeatedly on purpose at initial practice and unconsciously at a later practice. The learner continues the cycle in hula-hooping.

As a case study, we measured the actual relationship between phase difference [rad] and relative hoop height $[\mathrm{m}]$ to the waist for five experts. We considered experts to mean persons who could keep hula-hooping for a long time and who could sometimes adjust the hoop even if the vertical position changed. These experts all used the same hula-hoop and played with it freely for more than $10 \mathrm{~s}$. We monitored the trajectory of movements of both the waist and the hoop together using an optical motion capture system. Then, we calculated the phase difference. A typical example of performance of a player in one trial is illustrated in Figure 1 and the correlation coefficients were high $(r=-8.8$,

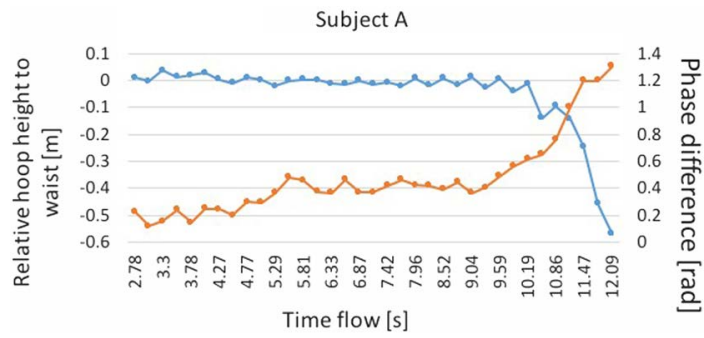

Figure 1. Example of Performance of a Player.

\section{Monitored spacing}

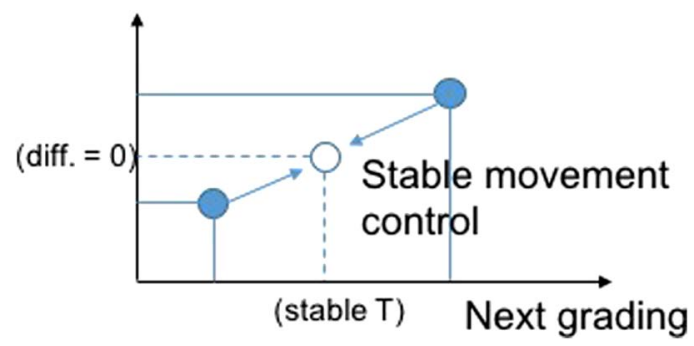

Figure 2. Controlling Direction from Positions of Getting Away.

where the mean cycle was 0.84 ).

From this figure, we discovered that the relative hoop height to the waist usually remained near zero. However, it fell as the phase difference rose. Specifically, until about $9 \mathrm{~s}$ from the start, the phase difference was a small fluctuation around 0.3. When the phase difference rose gradually to 0.5 or more, the relative hoop height fell. This indicated that the learner had to change the sense for keeping the phase difference between the waist and hoop movement.

\subsection{Principle of the Guide Method}

The feedback method reinforces the awareness of self-movement in the physical domain. In selecting the next grading degree with the monitored spacing degree, it is necessary to acquire a scheme of grading prediction during the practice. Figure 2 is a conceptual diagram of stable control in a simple harmonic movement.

If a spacing degree is less than zero, which is the reference criterion considering an off-set value, the guide suggesting the next timing of enforcing should be 


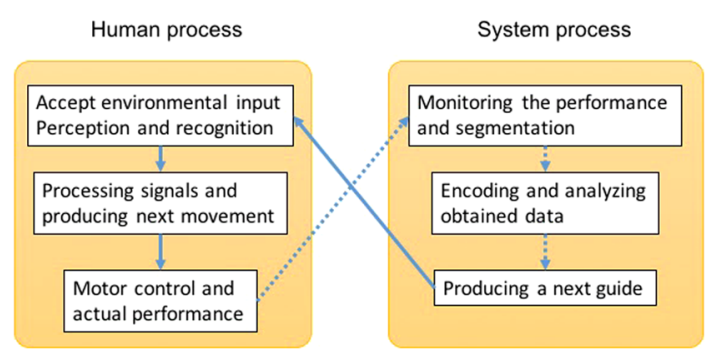

Figure 3. Human Process and an Associated System Process.

given sooner in order to make control more stable. If the monitored spacing degree is more than the criterion, the guide in this case should be given later so as to control the grading of the next monitoring phase in a counteraction.

The direction is understood from an ordinary control principle. However, simple feedback on an unmodified phase difference seems to remain off-set for some reasons; i.e. delayed feedback is always provided with one cycle difference. Therefore, this study introduces some correction values for the required time scale calibration.

\section{System Design}

\subsection{System Configuration}

Playing with a hula-hoop represents a kind of gross motor action. In supporting the skill for playing with the hula-hoop, it is necessary to monitor both movements of the body and of the hoop. In this study, we used an optical motion capture system, named Optitrack, to detect the body movement and integrated it with the associated trajectory of a hoop in a three-dimensional model. The reason we selected this monitoring device was based on some critical comparisons including:

a. the image processing techniques for movie images captured by a video camera takes a much longer time than is acceptable for the processing time; and

b. Kinect by Microsoft cannot capture the movement of a hoop because of its internal human model.

Figure 3 indicates an association between a human process flow and a system process flow during practice with our proposed system.

The system flow is a counterpart of the human process but its processing round is delayed by some period

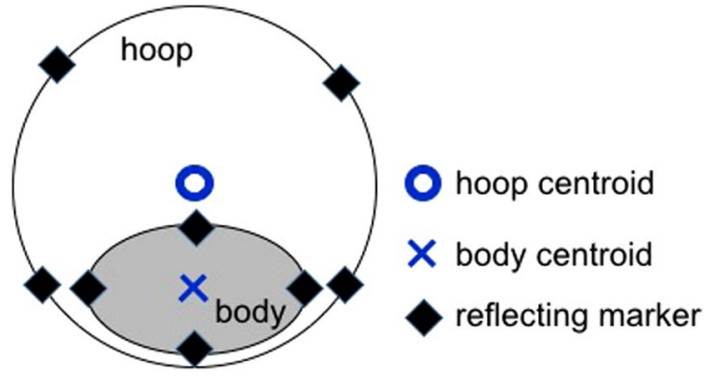

Figure 4. Relative Position of the Waist to the Hoop in a Top View.

relative to the human process. The motion capture system always monitors the human and the hoop movements together but it buffers the data until completion of the succeeding segment in getting the cycle unit. When the system succeeds in identifying both local maximum and minimum values, it calculates the next timing following the principle of this study. The timing is influenced by the expected spacing as the height of the hoop. As output of the system, sound and visual information are realized at the same time.

\subsection{Monitoring}

The capturing rate of the optical motion capture system is 100 [fps] as the default value, which is enough to monitor and create the wave form because even a fast player twirls the hoop approximately two times per second. The right-handed system is adopted for the threedimensional coordinates where $x$-coordinate is left-right to the body, the $y$-coordinate is vertical upward-downward and the $z$-coordinate is to the front-back of the body.

Figure 4 is a top view of the hoop and the player body inside it. The actual movement trajectory of the waist and the hoop is traced as its centroid, which is provided by monitoring software. Four markers are used to generate a rigid-body model in this context. This plane is composed of $x$ - and $z$-coordinates. The system identifies the movement on the plane in terms of the waist and hoop trajectory for which values in the $y$-coordinate are used for detecting the hoop position relatively. 


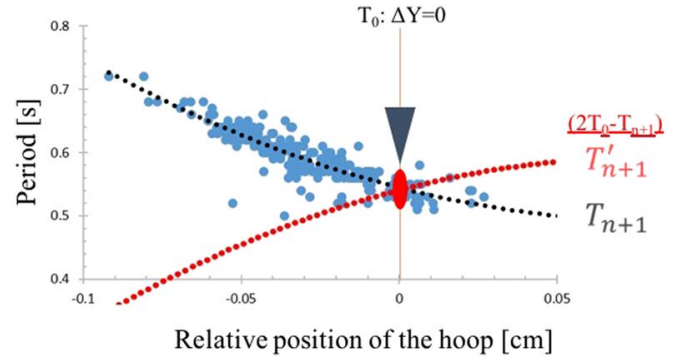

Figure 5. Prediction and Supporting Model.

\subsection{Analysis and Support}

Using data input from the motion capture system, our system analyzes all the stored data. The developed software provides the period of a hoop cycle, relative delayed time of the hoop movement for the waist and relative position as an average of the cycle. The procedures are summarized as follows;

(1) Relative position calculation (subtracts the waistposition from the hoop-position in the $z$-coordinate)

(2) Index detection of the local maximum of both waist and hoop positions

(3) Time difference calculation between the two indices in (2)

(4) Calculation of the period in the cycle of the hoop

(5) Calculation of the average value of the hoop position in (4)

(6) Repetition of procedures from (1) to (5)

All these data are stored to create a dynamic model from the viewpoint of supporting an approximate curve generation. A simple prediction model is made using a two-dimensional regression model based on the stored data, Figure 5.

The $x$-axis in Figure 5 indicates the relative position of the hoop against the waist while the $y$-axis indicates its period. Actual movement of one cycle is plotted by the dots. $T_{n+1}$ indicates the regression curve of the real trajectory. Suggestion model for the next movement is represented in $T_{n+1}^{\prime}$. When we get points in two dimensions in this figure, we can generate an approximate curve by regression analysis automatically. Using this, we calculate a supporting model in order to negate the prediction result. The closer the relative hoop position approaches zero, the shorter the period of the next

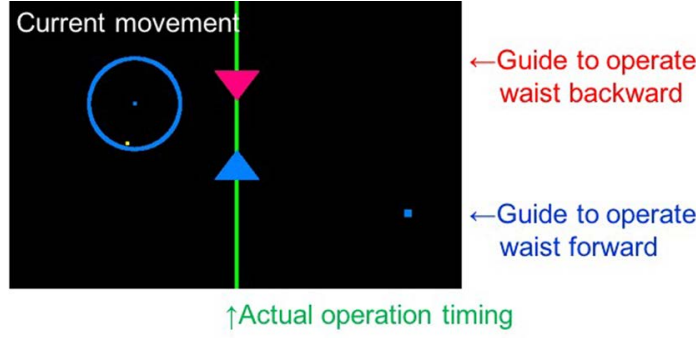

Figure 6. Visual Interface of the Proposal.

cycle becomes in this context. As a result, the support model tends to converge the movement to the position where the relative position of the hoop becomes zero.

The system mainly gives suggestions by sound, at a frequency of $880 \mathrm{~Hz}$. When the period is far from the reference point (the hoop is unsteady), the alarm sound is strong, otherwise it is weak. The sound is always produced but learners have a tendency not to notice it. In parallel, visual feedback is set up on a large screen on site, Figure 6.

\subsection{Feedback Implementation}

In the user interface we developed in this study, real-time movement of a hoop is shown on the left (Figure 6) where the centroid position is detected by the motion capture system and its radius is previously defined.

A learner can grasp the situation from the top view image on the fixed ground position on the circle, which is associated with the movement.

There are two small rectangles that come closer to each other in an animation movie, starting from the right to the left. Figure 6 shows one of them. Location of the rectangle above indicates backward movement of the waist while one below indicates waist-forward movement. The support model provides the timing where either one of the rectangles collides with the line in the center of the interface. When a rectangle aligns with the line, the animation is shown again from the right to the left cyclically. Two triangles on the center-line suggest how fast the waist should move at the appropriate timing. The upper one indicates the inclination of the waist in moving backwards while the lower one is for the inclination in moving forward every time a guide rectangle aligns with the line. All these visual guides are 


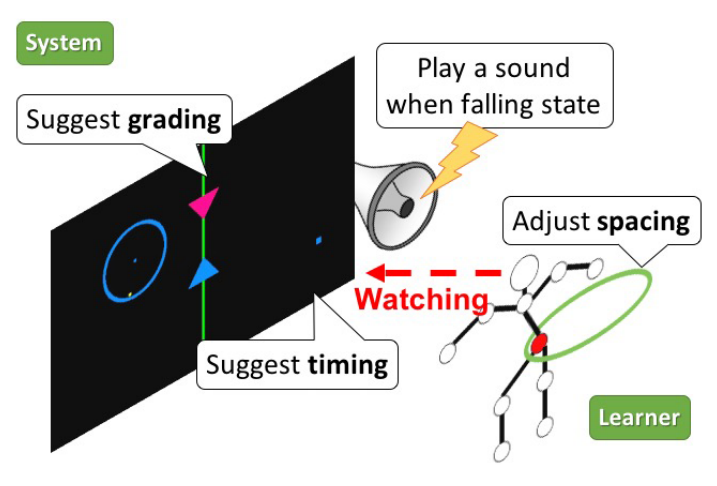

Figure 7. System Configuration and the Learner Process.

well associated with the sound feedback.

The configuration of the system and the learner's process are shown in Figure 7.

\section{Evaluation}

\subsection{Overview of the Experiment}

We conducted an experiment for the guide system evaluation in order to confirm the effect on our proposal. We wanted to investigate whether the proposal was effective for learning as well as whether it was a good periodical way of suggestion, considering both performance and recognition viewpoints. The number of volunteer subjects was 21 , and all were university students in their 20 's. They were healthy and had no physical limitations to playing with hula-hoop. Subjects gave their informed consent to participate in the study.

Concrete values of the evaluation were acquired for performance data on motion with respect to an objective viewpoint on spacing (Figure 7). Subjects were asked to answer a questionnaire for the subjective viewpoint. There were three conditions offered; (I) an environment following the proposal which suggested the next timing calculated dynamically, (II) an environment of periodic support for suggestion which was independent of monitored behavior and (III) an environment without any suggestion. A questionnaire, having contents dealing with subjective recognition of a relative hoop position, was distributed to subjects after the performance in every phase. The goal achievement was given openly and shared with subjects. They knew that the smaller difference between centroids of the waist
Table 1. Performance Summary in Pre-phase.

\begin{tabular}{|l|c|c|c|}
\hline & Group-A & Group-B & Group-C \\
\hline Ave. $[\mathrm{cm}]$ & -5.707 & -5.964 & -4.277 \\
\hline Within-group var. & 0.524 & 0.362 & 0.228 \\
\hline
\end{tabular}

and hoop made the performance response more stable.

There were three phases in the experiment; prephase, practice-phase and post-phase. In the pre-phase, all subjects rotated the hula-hoop freely without any feedback. In this phase, subjects tried to rotate the hoop three times steadily and monitoring the performance provided an average value of spacing degree. Subjects were divided into three homogeneous groups A, B and $\mathrm{C}$ from such viewpoints as average performance. When average performance is zero, this is the best because it indicates the hoop position is closest to the centroid of the waist. Both tests of Shapiro-Wilk for testing normality and Kruskal-Wallis for confirmation of the same distribution were carried out to confirm the groups were homogeneous. Three groups had seven members each. The values of average and within-group variance, which are based on pre-phase performance, are listed in Table 1.

In pre-phase with three trials, one day was assigned to every subject and three days were assigned for every subject in the practice-phase. Subjects did the experiment trial three times per day and answered a questionnaire after the trials in the practice-phase. Members in group-A used our proposed system whereas group-B members used a periodical suggestion system. Period for group-B was the average of the support model described in section 3.3. An actual value for each subject in group-B was previously determined where the spacing performance was approximately convergent in three trials. For example, a fixed value of phase difference in group-B was calculated as 0.32 . Feedback methods with both audio and visual information were the same but the way of determination of values was different between group-A and group-B. The best performance was when the average performance was zero, which means the relative position of the hoop is the same as the position of the waist. Therefore, the average value was calculated for checking the best performance. The difference between these groups was a dynamical adjustment presented for only group-A. Before starting the experiment, the meaning of animation movie and sound were explained to all subjects. Regarding group$\mathrm{C}$, they just played with the hula-hoop without any sug- 
gestion while the proposed system monitored their performance in the same way as in group-A.

We compared performance between pre-phase and post-phase. Recognition change was also investigated through the result of the questionnaire survey. We wanted to enable comparison between performance and the recognition change.

\subsection{Results}

\subsubsection{Performance Investigation}

Table 2 shows the post-phase performance as average values and standard deviation (S.D.). Table 3 indicates the difference of the absolute values in the postphase (Table 2) from those of the pre-phase (Table 1). The difference is given as the average and the standard deviation is also given. If the values became high, the performance was considered to have improved.

There are always individual variations in physical properties and characteristics. However, for the seven subjects of each group, we tried to carry out statistical tests and multiple comparisons among them.

We found no normality in obtained data from a statistical viewpoint. The Shapiro-Wilk test was applied for each group as for non-normality test. The actual values of groups-A, B and C were $p=0.002,0.088$ and 0.015 respectively with the significance level of $5 \%$. Two of the three groups had no normality and therefore we could not apply a parametric method in this case. As a result, the non-parametric test of Kruskal-Wallis was carried out on the resulting data in Table 3.

H0 (null hypothesis) produced no difference in improvement among groups. Our obtained data indicated that there was significant difference $(p=0.039$

Table 2. Performance Summary in Post-phase.

\begin{tabular}{|l|c|c|c|}
\hline & Group-A & Group-B & Group-C \\
\hline Ave. $[\mathrm{cm}]$ & -0.938 & -6.734 & -5.243 \\
\hline S.D. & 2.150 & 6.517 & 7.885 \\
\hline
\end{tabular}

Table 3. Performance Difference between Pre-phase and Postphase.

\begin{tabular}{|l|c|c|c|}
\hline & Group-A & Group-B & Group-C \\
\hline Diff. $[\mathrm{cm}]$ & 3.825 & -0.770 & -2.526 \\
\hline S.D. & 7.822 & 4.747 & 2.083 \\
\hline
\end{tabular}

$<0.050$ ), which meant rejection of H0. Consequently, multiple comparisons in the Steel-Dwass test was carried out. Only the pair of group-A and group-C was significant $(p=0.048<0.050)$ while other possibilities were not significant statistically (results in pairs of A-B and B-C were $p=0.762(>0.050)$ and $p=0.152 \quad(>0.050)$, respectively).

The performance data on the last day of the practice-phase and that of the post-phase was also tested from statistical viewpoint. We applied the one-way ANOVA, which indicated significance $(p=0.015<0.050)$ because the groups indicated normality $(p=0.596,0.346$ and $0.290(>0.050)$ by the Shapiro-Wilk test). Therefore, parametric analysis by the Turkey-Kramer test was carried out for multiple comparisons. As a result, only the pair of group-A and group-B indicated significant difference $(p=0.013<0.050)$ while other possibilities were $p=0.504$ and $0.123(>0.050)$, respectively.

\subsubsection{Recognition Investigation}

Subjects were requested to answer a questionnaire at every practice, which asked at what length separation distance they recognized the position of a hoop was away from the waist. Though the performance improvement led to objective outcomes, subjective change was also investigated in order to confirm the consciousness during the practice. When subjective data of the separation distance between the hoop and the waist were collected in average, the data were compared with the actual data monitored by the motion capture system.

Table 4 lists the average difference between prephase and post-phase in each group and the standard deviation (S.D.), which is the difference between prephase and post-phase in each group.

Recognition skill is one of the important factor to be focused on. Therefore, we gave the questionnaire and obtained results for the subjective position of the hoop, which were described in Section 4.1. We assumed the recognition error between these subjective values and observed with the training that real data were getting

Table 4. Recognition Difference between Pre-phase and Postphase.

\begin{tabular}{|l|c|c|c|}
\hline & Group-A & Group-B & Group-C \\
\hline Ave. Diff. [cm] & 5.274 & 1.016 & 0.472 \\
\hline S.D. & 7.307 & 3.970 & 2.208 \\
\hline
\end{tabular}


smaller than they were at the beginning. Therefore, the differences between pre-phase and post-phase errors were surveyed in Table 4. One-way ANOVA was carried out because the data followed a normality distribution, which was confirmed by the Shapiro-Wilk test. H0 (null hypothesis) showed no difference among groups in improvement due to consciousness of the hoop-distance from the waist. The results indicated no significant change $(p=0.214>0.050, F=3.554)$. The average of group-A was highest but the statistical result indicated not much difference.

\subsection{Discussion}

We carried out the experiment using the three systems; our proposed guide system (used by group-A), the alternative system of the periodical notification (used by group-B) and the control system of no suggestion (used by group-C). Only the pair of groups-A and $\mathrm{C}$ had any significant difference from the average performance improvement, which was from pre-phase to post-phase. Post-phase was the final stage of the experiment, and it took place after the different practices of the groups in three days. Therefore, the approach obviously contributed to the subjects' performance using the hula-hoop. However, the degree of improvement was not so different from that of a simple notification, which was based on the average-best performance monitored in several trials beforehand.

Group-A used our proposed system. Their data on average converged in a positive direction in all trials. The performance in group-B was similar in total but the data included both negative and positive changes. There seemed to be a significant difference on the last day of the support. Group-C showed no common tendency through the experiment.

Feedback effect was checked by a comparison between the last day of practice and the post-phase without feedback. Some members in experimental group-A increased their performance in the post-phase compared with the practice-phase and others were unchanged. Some members in group-B were unchanged but others fell in the post-phase.

With respect to the recognition change during the experiment, there was no statistical significance in any comparison of groups. There was no clear consciousness in the mind even though the performance improved. We want to make a further investigation on such difference in other research contexts. It seems this is a kind of difficulty in comprehension of the motorskill as tacit understanding.

\section{Concluding Remarks}

This paper proposed the guide system for learning support of a repetitive motor-skill. The concrete target in this study was training in spinning a hula-hoop. What learners acquired through the practice with our proposed system was to make a dynamic adjustment by way of parameter selection. We saw that performance was improved to some extent by using the system. At first, our work basically introduces the conventional theorybased concepts of timing, grading and spacing for the training environment. The designs of the audio and visual notifications are based on the two-dimensional regression model with time scale calibration.

We believe, the methodology in this paper is applicable to other similar domains. The conditions for application are simple; the target motor-skill includes a repetitive movement task done at the same place and the movement can be monitored by the system to create and analyze the wave form. Future applications of the system include tasks such as rope-skipping.

\section{Acknowledgement}

This work was supported by JSPS KAKENHI Grant Number JP15K01072.

\section{References}

(1) Guthrie, E. R.: The Psychology of Learning, Harper and Ro (1952).

(2) Schmidt, R. A.: "A Schema Theory of Discrete Motor Skill Learning”, Psychological Review, Vol. 82, No. 4, pp. 225260 (1975)

(3) Trowbridge, M. H. and Cason, H.: "An Experimental Study of Thorndike's Theory of Learning”, J. of General Psychology, Vol. 7, pp. 245-258 (1932).

(4) Johnson, H. W.: "Skill=Speed $\times$ Accuracy $\times$ Form $\times$ Adaptabi lity", Perceptual and Motor Skills, Vol. 13, pp. 163-170, Southern Universities Press (1961).

(5) Karungaru, S. G., Matsuura, K. and Gotoda, N.: "Feature Tracking Using Particle Filer in Rope Skipping for Gross Motor Skill Development”, Proc. of 2nd ICKE2016, (2016).

(6) Toyooka, H., Matsuura, K. and Gotoda, N.: "A Learning 
Support System Regarding Motion Trigger for Repetitive Motion Having an Operating Instrument", Proc. of 13th IADIS International Conference of CELDA, pp. 33-40 (2016).

(7) Fitts, P. M. and Possner, M. I.: Human Performance, Brooks and Cole (1967).

(8) Soga, M., Taki, H., Matsuda, N. et al.: "Skill Learning Support and a Framework for the Learning Support", Japanese Society for Artificial Intelligence, Vol. 20, No. 5,

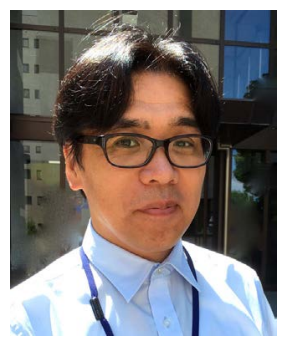

Kenji Matsuura received the M.E. and Ph.D. from Tokushima University in 1996 and 2002, respectively. He is currently a Professor at the Center for AIT, Tokushima University. His research interests include learning science, technology enhanced learning and human computer interaction. $\mathrm{He}$ is a member of JSiSE, IPSJ, JSET, JSAI and IEICE.

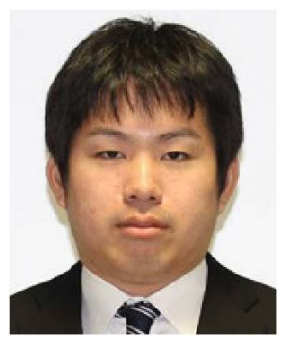

Hiroshi Toyooka received the B.E. and M.E. from Tokushima University in 2015 and 2017, respectively. He currently works as a system engineer at Glory LTD. His research interests include information science and knowledge engineering. pp. 533-540 (2005).

(9) Seyranian, A. P. and Belyakov, A. O.: "How to Twirl a Hula Hoop", American J. of Physics, Vol. 79, No. 7, pp. 712-715 (2011).

(10) Matsuura, K., Hamagami, K. and Yamada, K.: "A Learningsupport System on Repetitive Motor-skill: The Case of Hula-hoop," The Transactions of the Institute of Electronics, Information and Communication Engineers D, Vol. J98D, No. 1, pp. 61-70 (2015).

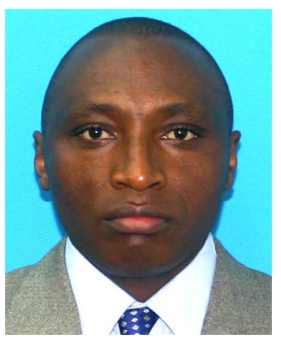

Stephen Karungaru received the M.E. and Ph.D. from Tokushima University in 2001 and 2004, respectively. He is currently a lecturer in the Graduate School of Science and Technology, Tokushima University. His research interests include pattern recognition, image processing and human recognition. He is a member of IEEJ and IEEE.

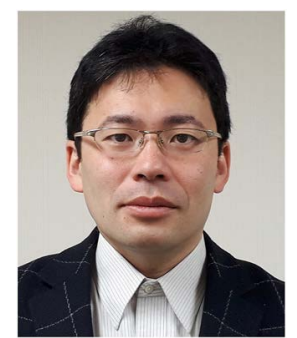

Naka Gotoda received the M.E. and $\mathrm{Ph}$.D. from Tokushima University in 2008 and 2010, respectively. He is currently an assistant professor of Kagawa University. His research interests include skill science. He is a member of IEICE, HIS, JSiSE and JSAI. 